Note

\title{
Growth in Acanthamoeba sp. and Antibiotic Susceptibility of Legionella micdadei Isolated from Hot Spring Water Samples
}

\author{
KATSUNORI FURUHATA ${ }^{1 *}$, KIKUMI OGIHARA ${ }^{1}$, RUMI OKUNO $^{2}$, \\ KENJI OONAKA ${ }^{1}$, AND MASAFUMI FUKUYAMA ${ }^{1}$ \\ ${ }^{1}$ School of Life and Environmental Science, Azabu University, \\ Sagamihara, Kanagawa 229-8501, Japan \\ ${ }^{2}$ Department of Microbiology, Tokyo Metropolitan Institute of \\ Public Health, Shinjuku, Tokyo 169-0073, Japan
}

Received 24 February, 2009/Accepted 15 July, 2009

\begin{abstract}
As part of an epidemiological study on legionellosis, we attempted to isolate Legionella spp. from hot spring water samples, and were able to isolate Legionella micdadei from $3(5.5 \%)$ of 55 samples. All of these isolates were able to grow within Acanthamoeba sp., suggesting that the isolates will be pathogens. We also confirmed that the K-2 strain from hot spring water grew in guinea pig monocytes. Sensitivity tests using 10 drugs showed that the isolates were most sensitive to imipenem, with the $\mathrm{MIC}_{90}$ of $0.032 \mu \mathrm{g} / \mathrm{ml}$, were least sensitive to minocycline, with the $\mathrm{MIC}_{90}$ of $4 \mu \mathrm{g} / \mathrm{ml}$, and were not sensitive to low amounts of other drugs.
\end{abstract}

Key words : Legionella micdadei / Growth in Acanthamoeba sp. / Antibiotic susceptibility/Hot spring water

A recent mass outbreak of legionellosis transmitted through hot spring water in Japan highlighted the importance of the hygienic management of bath water (Yabuuchi and Agata, 2004). Among the causative agents of legionellosis, a respiratory disease, Legionella pneumophila is well known, and extensive information is available on this bacterium (Edelstein and Meyer, 1980; Riffard et al., 1998; Albers et al., 2005; Amemura-Maekawa et al., 2008). On the other hand, a few cases of Legionella micdadei infection have been reported in the literature, but, to date, few studies have investigated the properties of this bacterium (Dowling et al., 1984; Koide et al., 1988; Goldberg et al., 1989; Bangsborg et al., 1991; Takiguchi et al., 1999). L. micdadei, a causative agent of legionellosis, was discovered by Tatlock (1944), and was referred to as the Tatlock agent. It was proposed as a new species, Tatlockia micdadei, by Garritty et al. (1980), but was later included in $L$.

*Corresponding author. Tel: +81-42-754-7111, Fax : +8142-754-6215. E-mail: furuhata (a) azabu-u.ac.jp micdadei (Hébert et al., 1980). In this study, we examined the pathogenicity and drug sensitivity of $L$. micdadei isolated from hot spring water.

Fifty-five test samples were collected into sterile polyethylene containers $(500 \mathrm{ml})$ in nine prefectures in Japan between May and October 2008, stored in a refrigerator until being cultured, and tested as follows.

Two hundred-milliliter samples were concentrated to $1 \mathrm{ml}$ by centrifugation at $6,000 \mathrm{rpm}$ for $30 \mathrm{~min}$. This concentrate was mixed with an equal volume of $0.2 \mathrm{M}$ $\mathrm{HCl}-\mathrm{KCl}$ solution $(\mathrm{pH} 2.2)$, and the mixture was incubated at room temperature for $15 \mathrm{~min}$. A $0.1-\mathrm{ml}$ aliquot of this mixture was spread over the surface of GVPC $\alpha$ agar medium (Merck Ltd. - Japan, Tokyo, Japan) with a Conradi stick, and cultured at $36{ }^{\circ} \mathrm{C}$ for 7 days. Subsequently, several colonies of each isolate suggestive of the genus Legionella were picked up, smeared onto a 2-compartment agar plate (Nikken Bio Medical Laboratory Inc., Kyoto, Japan) consisting of BCYE $\alpha$ and blood agar media, and subjected to pure culture and testing for cysteine requirement. In addition, strains that failed to grow in 
blood agar medium but grew in BCYE $\alpha$ agar medium alone were gram-stained, and long rod-shaped, gramnegative strains were presumed to belong to the genus Legionella. Next, strains were identified by latex agglutination (Kanto Chemical Co., Inc., Tokyo, Japan) and DNA-DNA hybridization (Kyokuto Pharmaceutical Industrial Co., Ltd., Tokyo, Japan).

Legionella isolates were tested for intra-amoebic growth as follows, according to the amoeba-agar method developed by Miyamoto et al. (2003). The surface of BCYE $\alpha$ agar was sufficiently dried, and 3 $\mathrm{ml}$ of a suspension of the Acanthamoeba JAC/E1 strain that had been cultured in PYGC medium at 30 ${ }^{\circ} \mathrm{C}$ for 7 days was dropped onto and spread over the entire surface. The agar plate was incubated at 30 ${ }^{\circ} \mathrm{C}$ for $3 \mathrm{~h}$ to allow the amoebae to adhere firmly to it, and then the excess suspension was removed. Samples were applied simultaneously to this medium (referred to as amoeba-agar below) and BCYE $\alpha$ medium, and were cultured at $30^{\circ} \mathrm{C}$ for 7 days. Samples that formed colonies in both culture media were regarded as being able to grow in amoebae. The clinical isolate L. pneumophila IID 5232 and attenuated strain L. pneumophila 25D (Horwitz, 1987) were used as positive and negative controls, respectively.

One milliliter of peripheral blood was collected from a guinea pig, and treated with heparin. This blood sample was infected with $L$. micdadei strain $\mathrm{K}-2$ by the addition of $100 \mu \mathrm{l}$ of the bacterial suspension. After $24 \mathrm{~h}$, blood smears were prepared, stained by the Gimenez method, and observed for L. micdadei within monocytes.

Drug sensitivity tests were performed using Etest (Aska Diagnostics Inc., Tokyo) according to the attached technical guide. The drugs tested were erythromycin (EM), clarithromycin (CAM), azithromycin (AZM), minocycline (MINO), levofloxacin (LVFX), ciprofloxacin (CPFX), piperacillin (PIPC), imipenem (IPM), gentamicin (GM), and rifampicin (RFP) (10 drugs). The bacterial suspension of each test strain $(0.5 \mathrm{ml})$ was dripped on BCYE $\alpha$ agar medium (Merck Ltd. -Japan, Tokyo) (60 ml in a $150-\mathrm{mm}$ dish (Corning Inc., USA)) and smeared over the surface using a Conradi stick, and an Etest strip was closely attached onto the medium. The plates were cultured at $30^{\circ} \mathrm{C}$ for 5 days, and the growth inhibition zone formed around the strip was observed. Minimum Inhibitory Concentration (MIC) was judged by macroscopically reading the gradation at which the end of the growth inhibition zone and the strip crossed.

L. micdadei was successfully isolated from 3 $(5.5 \%)$ of the 55 samples of hot spring water (TABLE 1). Of these, samples F-1 and F-2 were collected from swimming pool and indoor hot spring water, respectively, from the $T$ spa facility in Hiroshima Prefecture, and were simple alkaline spring water $(\mathrm{pH}$ 9.1). The numbers of bacteria detected in these samples were $6.0 \times 10$ and $5.0 \times 10 \mathrm{CFU} / 100 \mathrm{ml}$, respectively. Sample K was collected from open-air hot spring water from the $U$ spa facility in Kanagawa Prefecture, and was sodium-chloride spring water with a sodium ion concentration of $10,920 \mathrm{mg} / \mathrm{L}(\mathrm{pH}$ 7.4), yielding $5.0 \times 10 \mathrm{CFU} / 100 \mathrm{ml}$. Furthermore, $L$. pneumophila was isolated from 14 samples (25.5\%). However, L. micdadei was not isolated from these samples.

Twenty-seven L. micdadei strains (12 F-1 and F-2 strains each and three $\mathrm{K}$ strains) isolated from hot spring water formed colonies on amoeba-agar medium, as in the positive control. These colonies did not differ at all from those formed on BCYE $\alpha$ agar medium used as a control, suggesting that these strains grew in Acanthamoeba sp. cells.

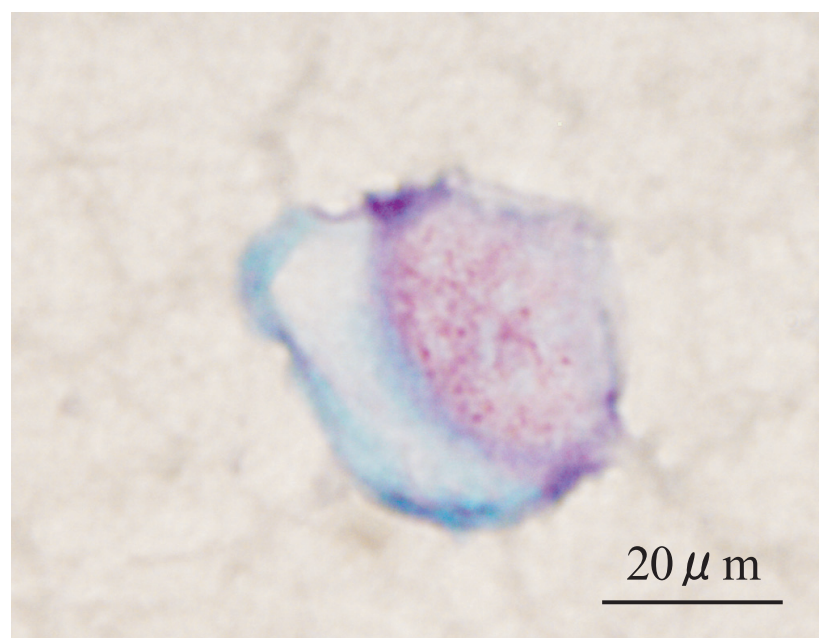

FIG. 1. Giménez staining of L. micdadei in guinea pig monocytes.

TABLE 1. Isolation of L. micdadei from hot spring water samples.

\begin{tabular}{ccccccc}
\hline Samples & Prefecture & Facility & Sampling sites & $\mathrm{pH}$ & Quality & $\begin{array}{c}\text { Viable numbers } \\
(\mathrm{CFU} / 100 \mathrm{ml})\end{array}$ \\
\hline $\mathrm{F}-1$ & Hiroshima & $\mathrm{T}$ & $\begin{array}{c}\text { Swimming pool } \\
\text { Indoor-spa }\end{array}$ & 9.1 & Simple alkaline & $\begin{array}{c}6.0 \times 10 \\
5.0 \times 10\end{array}$ \\
\hline $\mathrm{F}-2$ & Kanagawa & $\mathrm{U}$ & Open-air bath & 7.9 & Sodium chloride & $5.0 \times 10$ \\
\hline
\end{tabular}


TABLE 2. Susceptibility of $L$. micdadei isolated from hot spring waters to antimicrobial agents.

\begin{tabular}{|c|c|c|c|c|c|c|c|c|c|c|c|c|c|c|c|c|}
\hline \multirow{2}{*}{$\begin{array}{l}\text { Antimicrobial } \\
\text { agents }\end{array}$} & \multicolumn{14}{|c|}{$\operatorname{MIC}(\mu \mathrm{g} / \mathrm{ml})$} & \multirow{2}{*}{$\mathrm{MIC}_{50}$} & \multirow{2}{*}{$\mathrm{MIC}_{90}$} \\
\hline & $<0.002$ & 0.002 & 0.004 & 0.008 & 0.016 & 0.032 & 0.064 & 0.125 & 0.25 & 0.5 & 1 & 2 & 4 & 8 & & \\
\hline Erythromycin & & & & & & & & 4 & 9 & 11 & 3 & & & & 0.5 & 1 \\
\hline Clarithromycin & & & & & & & 1 & 10 & 11 & 4 & 1 & & & & 0.25 & 0.5 \\
\hline Azithromycin & & & & & 1 & 1 & 2 & 12 & 7 & 1 & 3 & & & & 0.125 & 1 \\
\hline Minocycline & & & & & & & & & & 1 & 6 & 14 & 5 & 1 & 2 & 4 \\
\hline Levofloxacin & & & & & & & & & 2 & 24 & 1 & & & & 0.5 & 0.5 \\
\hline Ciprofloxacin & & & & & & & & & & 15 & 12 & & & & 0.5 & 1 \\
\hline Piperacillin & & & & & 19 & 2 & 3 & 1 & 2 & & & & & & 0.016 & 0.125 \\
\hline Imipenem & 4 & 1 & 8 & 3 & 7 & 2 & 2 & & & & & & & & 0.008 & 0.032 \\
\hline Gentamicin & & & & & & & & 9 & 8 & & 3 & 6 & 1 & & 0.25 & 2 \\
\hline Rifampicin & & & 1 & & 2 & 13 & 10 & 1 & & & & & & & 0.032 & 0.064 \\
\hline
\end{tabular}

$\mathrm{n}=27$

Guinea pig monocytes in peripheral blood were infected with the K-2 strain of L. micdadei, and stained by the Giménez method. The stained image is shown in FIG. 1. Thus, it was confirmed that L. micdadei grew in the cytoplasm of monocytes, compressing the nuclei to the periphery. In addition, the growth of L. micdadei resulted in the destruction of some monocytes in the blood smear.

TABLE 2 shows the susceptibility of the 27 test strains to 10 drugs. The MIC values of EM, CAM, AZM, MINO, LVFX, CPFX, PIPC, IPM, GM, and RFP were in the ranges of $0.125-1,0.064-1,0.016-1,0.5-$ $8,0.25-1,0.5-1,0.016-0.25,<0.002-0.064,0.125-4$ and $0.004-0.125 \mu \mathrm{g} / \mathrm{ml}$, respectively. The MICs of 8 of the 10 drugs tested showed a unimodal peak, but those of IPM and GM exhibited bimodal peaks at $0.004,0.016 \mu \mathrm{g} / \mathrm{ml}$ and $0.125,2 \mu \mathrm{g} / \mathrm{ml}$, respectively. The MICs of all drugs for the test strains were $8 \mu \mathrm{g} / \mathrm{ml}$ or less; thus, no strains showed a particularly low sensitivity.

In terms of $\mathrm{MIC}_{90}$ values, the isolates were most sensitive to IPM at $0.032 \mu \mathrm{g} / \mathrm{ml}$, followed by RFP $(0.064 \mu \mathrm{g} / \mathrm{ml})$, PIPC $(0.125 \mu \mathrm{g} / \mathrm{ml}), C A M$ and $\operatorname{LVFX}(0.5 \mu \mathrm{g} / \mathrm{ml}), \mathrm{EM}, \mathrm{AZM}$ and CPFX $(1 \mu \mathrm{g} / \mathrm{ml})$, GM $(2 \mu \mathrm{g} / \mathrm{ml})$, and MINO $(4 \mu \mathrm{g} / \mathrm{ml})$. The sensitivities of the isolates to RFP, PIPC, CAM/ LVX, EM/AZM/CPFX, GM, and MINO were 2, 4, 16, 32, 64, and 128 times lower than that to IPM, to which the isolates were most sensitive (TABLE 2).

In this study, we isolated L. micdadei from hot spring water, and found that the bacterium inhabits hot spring water in Japan, although the rate of isolation was as low as 5.5\% (3 in 55 samples). In a nationwide study in 2003 (Furuhata et al., 2004a), we isolated L. micdadei in only $2(0.3 \%)$ of 710 samples, and the majority of the isolates belonged to $L$. pneumophila. In this study, two of the three samples, from which $L$. micdadei was isolated, were simple alkaline spring water with $\mathrm{pH}$ 9.1, and the remaining one sample was sodium-chloride spring water with a high sodium-ion concentration of $10,920 \mathrm{mg} / \mathrm{L}$. Sheehan et al. (2005) isolated L. micdadei from a eukaryotic algal mat community in a $\mathrm{pH} 2.7$ geothermal stream in the Yellowstone National Park. Thus, it became clear that $L$. micdadei is distributed widely in aquatic environments that vary greatly in water quality.

To investigate the pathogenicity of $L$. micdadei from hot spring water, we examined its ability to grow intracellularly in Acanthamoeba sp. This method was developed by Miyamoto et al. (2003), and has been used to evaluate the pathogenicity of bacteria of the genus Legionella (Albers et al., 2005). The bacteria of the genus Legionella can grow intracellularly in protists that phagocytize bacteria, showing an ability to grow in human and animal macrophages. This property of the bacterium is reportedly important in considering the pathogenicity of the genus Legionella. Using a similar amoeba plate test on an agar containing Acanthamoeba castellanii, Albers et al. (2005) analyzed the gene expression in $L$. pneumophila. In this study, we confirmed that all $L$. micdadei isolates from the hot spring water samples were able to grow in Acanthamoeba sp., which strongly suggests that all isolates are pathogenic. Although the genes responsible for the pathogenicity of $L$. micdadei have not been identified, Morozova et al. (2004) reported that L. micdadei has genes analogous to the $\mathrm{icm} / \mathrm{dot}$ genes of $L$. pneumophila. Although they examined the presence or absence of analogous genes by Southern hybridization, they did not clarify whether these genes were involved in intracellular growth.

Sensitivity tests against 10 drugs showed that these isolates were most sensitive to IPM and RFP, with an $\mathrm{MIC}_{90}$ of $0.064 \mu \mathrm{g} / \mathrm{ml}$, and were not sensitive to low amounts of other drugs. In a previous study (Furuhata et al., 2004b), drug sensitivity tests also 
showed that $L$. pneumophila isolates from hot spring water were most sensitive to RFP, with an $\mathrm{MIC}_{90}$ of $0.125 \mu \mathrm{g} / \mathrm{ml}$. Edelstein and Meyer (1980) also tested the drug sensitivity of 33 clinical isolates of $L$. pneumophila, and reported that the MIC values of RFP were distributed between 0.025 and $0.125 \mu \mathrm{g} /$ $\mathrm{ml}$, indicating high-level sensitivity to RFP, similar to the results of this study.

Murakami et al. (2001) measured the MICs of various agents against clinical strain-derived $L$. pneumophila in Japan using the $\mathrm{E}$ test. The results did not show any drug-resistant bacteria. $L$. pneumophila was highly sensitive to macrolides and RFP, as reported in other countries

Thus, we considered that the L. micdadei isolates from hot spring water were pathogenic, and highly sensitive to IPM and RFP. Further studies are needed to identify the genes responsible for the pathogenicity of $L$. micdadei and to follow the changes in the drug sensitivity regarding the emergence of drug-resistant bacteria.

\section{ACKNOWLEDGEMENT}

This study was partially supported by a project grant (Start-Up Support for the Matching Fund Subsidy for Private Universities, 2007-2008) awarded by the Azabu University Research Services Division.

\section{REFERENCES}

Albers, U., Reus, K., Shuman, H.A., and Hilbi, H. (2005) The amoebae plate test implicates a paralogue of $/ p x B$ in the interaction of Legionella pneumophila with Acanthamoeba castellanii. Microbiol., 151, 167-182.

Amemura-Maekawa, J., Kura, F., Bin, C., Suzuki-Hashimoto, A., Ichinose, M., Endo, T., and Watanabe, H. (2008) Distinct difference of fla A genotypes of Legionella pneumophila between isolates from bath water and cooling tower water. Microbiol. Immunol., 52, 460-464.

Bangsborg, J.M., Cianciotto, N.P., and Hindersson, P. (1991) Nucleotide sequence analysis of the Legionella micdadei mip gene, encoding a 30-kilodalton analog of the Legionella pneumophila mip protein. Infect. Immun., 59, 3836-3840.

Dowling, J.N., Pasculle, A.W., Frola, F.N., Zaphyr, M.K., and Yee, R.B. (1984) Infections caused by Legionella micdadei and Legionella pneumophila among renal transplant recipients. J. Infect. Dis., 149, 703-713.

Edelstein, P.H., and Meyer, R.D. (1980) Susceptibility of Legionella pneumophila to twenty antimicrobial agents. Antimicrob.Agents Chemother., 18, 403-408.

Furuhata, K., Hara, M., Yoshida, S., and Fukuyama, M. (2004a) Distribution of Legionella spp. in hot spring baths in Japan (in Japanese). J.J.A.Inf. D., 78, 710-716. Furuhata, K., Hara, M., Fukuyama, M., and Yoshida, S. (2004b) Antibiotic sensitivity of Legionella pneumophila strains isolated from hot spring bath waters in Japan (in Japanese). Bokin Bobai, 32, 343-347.

Garrity, G.M., Brown, A., and Vickers, R.M. (1980) Tatlockia and Fluoribacter: two new genera of organisms resembling Legionella pneumophila. Int.J.Sys.Bacteriol., 30, 609-614.

Goldberg, D.J., Wrench, J.G., Collier, P.W., Emslie, J.A., Fallon, R.J., Forbes, G.I., McKay, T.M., Macpherson, A.C., Markwick, T.A., and Reid, D. (1989). Lochgoilhead fever: outbreak of non-pneumonic Legionellosis due to Legionella micdadei. Lancet, 316-318.

Hébert, G.A., Steigerwalt, A.G., and Brenner, D.J. (1980) Legionella micdadei species nova: Classification of a third species of Legionella associated with human pneumonia. Curr.Microbiol., 3, 225-257.

Horwitz, M.A. (1987). Characterization of a virulent mutant Legionella pneumophila that survive but do not multiply within human monocytes. J. Exp. Med., 166, 1310-1328.

Koide, M., Minamide, M., Nukina, M., Nakanishi, H., Kamiki, T., Ishikawa, T., Nagai, K., Umeda, F., Shirane, H., and Saitoh, A. (1988) Pneumonia caused by Legionella micdadei in a fatal case and its bacteriological characteristics (in Japanese). J.J.A.Inf.D., 62, 1-6.

Miyamoto, H., Taniguchi, H., and Yoshida, S. (2003). A simple qualitative assay for intracellular growth of Legionella pneumophila within Acanthamoeba culbertsoni (in Japanese). J.J.A.Inf.D., 77, 343-345.

Murakami, H., Matsumoto, T., Kobayashi, T., Isogai, K., Kashitani, F., Furuya, N., Tateda, K., and Yamaguchi, K. (2001) Susceptibility testing of clinical strains of Legionella spp. Isolated in Japan (in Japanese). J.J.A.Inf.D., 75, 1-6.

Morozova, I., Qu, X., Shi, S., Asamani, G., Greenberg, J.E., Shuman, H.A., and Russo, J.J. (2004) Comparative sequence analysis of the $\mathrm{icm} / \mathrm{dot}$ gene in Legionella. Plasmid, 51, 127-147.

Riffard, S., Presti, F.L., Vandenesch, F., Forey, F., Reyrolle, M., and Etienne, J. (1998) Comparative analysis of infrequent-restriction-site PCR and pulsed-field gel electrophoresis for epidemiological typing of Legionella pneumophila serogroup 1 strains. J.Clin.Microbiol., 36, 161-167.

Sheehan, K.B., Henson, J.M., and Ferris, M.J. (2005). Legionella species diversity in an acidic biofilm community in Yellowstone National Park. Appl.Environ.Mirobiol., 71, 507-511.

Takiguchi, Y., Nakamura, M., Aotsuka, N., Koseki, H., Terano, T., and Hirai, A. (1999). Bilateral pleuritis caused by Legionella micdadei. Internal Medicine, 38, 369-371.

Tatlock, H. (1944) A rickettsia-like organism recovered from guinea pigs. Proc.Soc.Exp.Biol.Med., 57, 95-99.

Yabuuchi, E., and Agata, K. (2004) An outbreak of Legionellosis in a new facility of hot spring bath in Hiuga city (in Japanese). J.J.A.Inf.D., 78, 90-98. 